\title{
Hard X-ray Surveys with coded mask telescopes
}

\author{
A. Segreto*, G. Cusumano, V. La Parola, T. Mineo, V. Mangano, P. Romano \\ INAF-IASFPa \\ Via U. La Malfa 153 \\ I-90146 Palermo \\ Italy \\ *E-mail: segreto@ifc.inaf.it

\section{Ferrigno} \\ Institut für Astronomie und Astrophysik Tübingen (IAAT) \\ and \\ ISDC Data Centre for Astrophysics \\ Chemin dŠÉcogia 16 \\ CH-1290 Versoix \\ Switzerland
}

Two coded mask telescopes are currently observing the hard X-ray sky: INTEGRAL/ISGRI and Swift/BAT. Their properties and pointing strategies complement each other: while ISGRI provides a better spatial resolution BAT has a much larger field of view. To analyze in a fast and efficient way the public archives of these two instruments, we have developed an imaging software suitable for both. We present some performance of the software and we show how the two archives can be fruitfully combined to reach a deeper survey sensitivity.

7th INTEGRAL Workshop

September 8-11 2008

Copenhagen, Denmark

${ }^{*}$ Speaker. 


\section{Introduction}

Hard X-ray (> $10 \mathrm{keV}$ ) and gamma ray sky images can be obtained with coded mask imagers (Skinner 1995) that record the shadow of a mask containing a transparent/opaque pattern projected onto the detector by all emitting sources of a large region of sky. Two coded mask imagers are currently observing the sky in the hard X-ray domain: the INTEGRAL Soft Gamma-Ray Imager (ISGRI) [1] on board INTEGRAL satellite and the Burst Alert Telescope (BAT) onboard the Swift satellite [2].

The INTEGRAL-ISGRI telescope is designed with a cyclic mask pattern, and is characterized by a FOV of $9^{\circ} \times 9^{\circ}$ (fully coded), fine imaging (12 arcmin FWHM) operating in the $15 \mathrm{keV}-$ $500 \mathrm{keV}$ energy range. Swift-BAT is designed with a pseudo-random mask pattern and is characterized by a large FOV, 1.4 steradian (half coded), designed to provide critical GRB triggers with 4-arcmin errorbox in the $15-150 \mathrm{keV}$ energy range and covering a fraction between 50 and $80 \%$ of the sky every day.

In order to fully exploit both the ISGRI and BAT survey data through a systematic search for new hard X-ray sources, we have developed a dedicated software, the BATIMAGER [8] that allows to analyze these two datasets working coherently on both and to easily combine their results.

\section{The code}

The BATIMAGER software has been designed to generate cleaned all-sky mosaics in a very efficient and fast way, starting directly from the raw ISGRI or BAT observation data.

At variance with respect to standard software package distributed ISGRI and BAT teams, where final mosaics products are obtained by application of several separate programs in a pipeline, in our software all processing steps, from shadowgram accumulation to mosaicking, have been integrated in one single highly optimized program, thus obtaining a large saving of CPU load and a considerable reduction in processing times.

The software performs shadowgram accumulation, cross-correlation with the coded mask pattern, accurate modeling of the non-uniform background distribution, correction of the instrumental systematics, correction for the cross-contamination between sources, skymap generation, skymap projection, mosaicking and fine localization and identification of hard X-ray sources. Moreover, beside all-sky mosaic images, the software produces also light curves and time resolved spectra for all the X-ray sources in the field of view.

To obtain a reduction of systematic effects and the improvement of the detection sensitivity, in our software we have devoted particular attention to the modeling of the coded mask pattern, of the detector background spatial variations, and of the pixel detection efficiencies.

A more detailed description of the software can be found in [8]; here we show some examples of performance of this code in terms of position reconstruction and imaging capability for both ISGRI and BAT instruments.

\section{Performance}

Fig. 1 shows a comparison of the Galactic center region obtained with Swift-BAT and INTEGRALISGRI showing the different spatial resolution of these two instruments. As evident, crowded fields 
are very well resolved testifying that BATIMAGER is able to produce sky images with the intrinsic spatial resolution of these two coded mask telescopes.
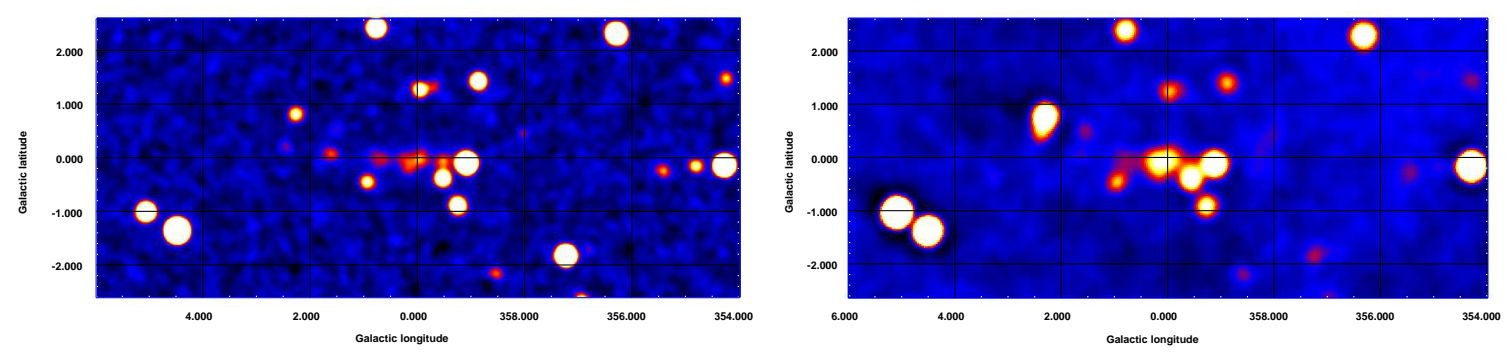

Figure 1: The Galactic center region (15-150 keV), as seen by ISGRI (left image) and BAT (right image)

In Fig. 2, the left panel shows the 90\% containment radius vs. detection significance for the two telescopes. To take into account the residual systematic errors in reconstructed images, the source detection significance is obtained as the ratio between the reconstructed source flux and the standard deviation measured in small region of the reconstructed sky images around the source position. The radius containment vs. the detection significance is well described by a power-law plus a constant model for both telescopes.

In the right panel of Fig. 2 it is shown an example of the statistical distribution of significance in the BAT all-sky mosaic reconstructed with our software. Apart from the positive tail due to the presence of sources, the distribution is well described by a gaussian with zero mean and unitary standard deviation.
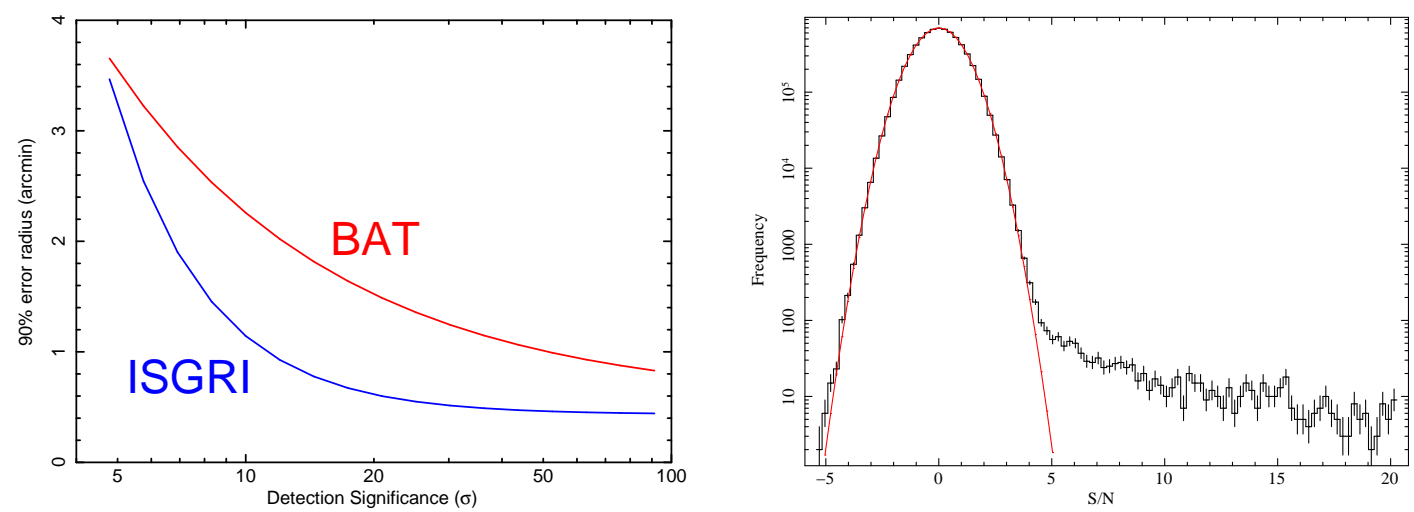

Figure 2: Left panel shows the mosaic localization 90\% error radius obtained with BATIMAGER. In the right panel an example of the statistical distribution of significance values in the BAT all-sky mosaic.

Figure 3 (left panel) shows the sky coverage achieved with 39 months of Swift-BAT survey and with ISGRI data available to date. The BAT sky coverage is much more uniform than the ISGRI one because of the much more uniform distribution of SWIFT spacecraft pointing directions with respect to INTEGRAL. In the same figure (right panel), we show the improvement in the survey sensitivity obtained when the BAT and ISGRI all-sky mosaics are combined. 

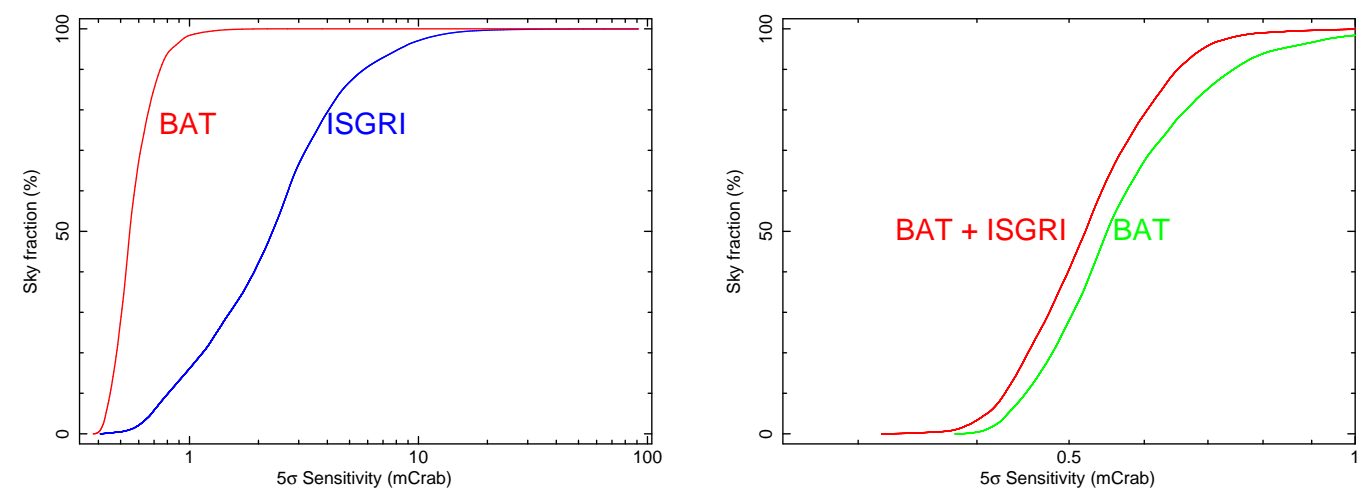

Figure 3: The left image shows the sky coverage fraction obtained from BAT and ISGRI all-sky mosaics separately. The sensitivity has been computed taking into account the residual systematic effects in image reconstruction, which become important for long exposures in complex fields. The right image shows the improvement in the survey sensitivity obtained combining the all-sky mosaics of these two instruments.

\section{Summary}

We report on our software, the BATIMAGER, developed in the frame of the Palermo Hard X-ray survey project. The software has been designed for a fast and optimal exploitation of both the BAT and ISGRI data, allowing to easily combine the surveys of these two coded mask instruments to reach a deeper sensitivity.

Some preliminary performance obtained with our software can be found in [6] and a more detailed description in [8]. The first results on new hard X-ray emitters discovered in the BAT survey data are presented in [4] [3] and the results on the search for unidentified sources in [5], and [7].

\section{References}

[1] P. Ubertini et al., IBIS: The Imager on-board INTEGRAL, A\&A 411 (L131) 2003

[2] S. D. Barthelmy et al., The Burst Alert Telescope (BAT) on the SWIFT Midex Mission, Space Science Reviews, 2005, 120, 143

[3] G. Cusumano, V. La Parola, A. Segreto, V. Mangano, C. Ferrigno, P. Romano, T. Mineo, S. Campana, G. Chincarini, P. Giommi, A. Moretti, G. Tagliaferri, The Palermo Swift-BAT hard X-ray catalogue.II. Results after 39 months of sky survey, 2009, A\&A, submitted

[4] G. Cusumano et al., New hard X-ray emitters discovered by BAT, 2009, in proceedings of 7th INTEGRAL Workshop, PoS (Integral08) 134.

[5] V. Mangano et al., Hard X-ray search for unidentified EGRET sources in the BAT and ISGRI survey archives, 2009, in proceedings of 7th INTEGRAL Workshop, PoS (Integral 08 ) 133.

[6] V. La Parola et al., Detection of ISGRI sources in 3 years all sky survey 2009, in proceeding of 7th INTEGRAL Workshop, PoS (Integra108) 132.

[7] B. Sbarufatti et al., Search for Roma-BZCAT Blazars in the Palermo BAT Survey archive, 2009, in proceeding of 7th INTEGRAL Workshop, PoS (Integral08) 069.

[8] A. Segreto, G. Cusumano, C. Ferrigno, V. La Parola, V. Mangano, T. Mineo, P. Romano, The Palermo Swift-BAT Survey. I. Methodology, 2009, A\&A, submitted 